\title{
The quality of Australian Indigenous primary health care research focusing on social and emotional wellbeing: a systematic review
}

\section{Sara Farnbach ${ }^{a, b, i}$, Anne-Marie Eades ${ }^{a, b}$, Jamie K Fernandoc ${ }^{c}$, Josephine D Gwynn',e, Nick Glozier ${ }^{f, g}$ and Maree L Hacketta,b,h}

a The George Institute for Global Health, UNSW, Sydney, Australia

b School of Medicine, University of Sydney, NSW, Australia

c School of Medicine and Public Health, University of Newcastle, NSW, Australia

a Poche Centre for Indigenous Health, University of Sydney, NSW, Australia e Faculty of Health Sciences, University of Sydney, NSW, Australia

${ }^{\dagger}$ Brain and Mind Centre, University of Sydney, NSW, Australia

g Central Clinical School, University of Sydney, NSW, Australia

h Faculty of Health and Wellbeing, University of Central Lancashire, Preston, UK

'Corresponding author: sfarnbach@georgeinstitute.org.au

\section{Article history}

Publication date: October 2017

Citation: Farnbach S, Eades AM,

Fernando JK, Gwynn JD, Glozier N, Hackett ML. The quality of Australian Indigenous primary health care research focusing on social and emotional wellbeing: a systematic review. Public Health Res Pract. 2017;27(4):e27341700.

https://doi.org/10.17061/phrp27341700

\section{Key points}

- More examples are needed of Indigenous-focused health research that are scientifically robust and acceptable to the community

- A range of research designs is used depending on the collaboration, community perspectives and the study aim

- Participatory action research can inform localised interventions and research designs, including randomised designs

- Processes that are culturally sensitive may improve community acceptance. These include two-way learning, participatory, social-ecological and phenomenological approaches

- Research should produce beneficial community-level outcomes

\section{Abstract}

Objectives and importance of the study: Primary health care research focused on Aboriginal and Torres Strait Islander (Indigenous) people is needed to ensure that key frontline services provide evidence based and culturally appropriate care. We systematically reviewed the published primary health care literature to identify research designs, processes and outcomes, and assess the scientific quality of research focused on social and emotional wellbeing. This will inform future research to improve evidence based, culturally appropriate primary health care.

Study type: Systematic review in accordance with PRISMA and MOOSE guidelines.

Methods: Four databases and one Indigenous-specific project website were searched for qualitative, quantitative and mixed-method published research. Studies that were conducted in primary health care services and focused on the social and emotional wellbeing of Indigenous people were included. Scientific quality was assessed using risk-of-bias assessment tools that were modified to meet our aims. We assessed community acceptance by identifying the involvement of community governance structures and representation during research development, conduct and reporting. Data were extracted using standard forms developed for this review.

Results: We included 32 articles, which reported on 25 studies. Qualitative and mixed methods were used in 18 studies. Twelve articles were judged as high or unclear risk of bias, four as moderate and five as low risk of bias. Another four studies were not able to be assessed as they did not align with the risk-of-bias tools. Of the five articles judged as low risk of bias, two also had high community acceptance and both of these were qualitative. One used a phenomenological approach and the other combined participatory action research with a social-ecological perspective and incorporated 'two- 
way learning' principles. Of the 16 studies where a primary outcome was identified, eight aimed to identify perceptions or experiences. The remaining studies assessed resources, or evaluated services, interventions, programs or policies. We were unable to identify primary outcomes in eight studies.

Conclusion: Conducting Indigenous-focused primary health care research that is scientifically robust, culturally appropriate and produces communitylevel outcomes is challenging. We suggest that research teams use participatory, culturally sensitive approaches and collaborate closely to plan and implement high-quality research that incorporates local perspectives. Research should result in beneficial outcomes for the communities involved.

\section{Introduction}

Health research that focuses on Aboriginal and Torres Strait Islander (Indigenous) people is needed to ensure that the healthcare provided is evidence based and culturally appropriate. Concerns about this type of research arise from the perception that, despite the large amount of research completed, community-level benefit ${ }^{1}$ and improvements in health outcomes are limited. ${ }^{2}$ To guide Indigenous-focused research, a set of guidelines for ethical conduct in Indigenous health research (Values and ethics) was developed. ${ }^{3}$ This document guides researchers and ethics committees on the conduct of culturally appropriate, community-acceptable research.

Conducting research that is culturally appropriate and acceptable to the Indigenous community where it is being completed may require modification of traditional research designs and processes. For example, use of participatory action research designs ${ }^{4}$ or research processes that involve extensive community consultation ${ }^{5}$ may mean that traditional approaches need to be adapted. Increasingly, Indigenous research methods and designs are being used. ${ }^{6}$ When conducting research that is culturally appropriate, researchers must balance using culturally appropriate methods with the need for research that is of high scientific quality.

The term 'social and emotional wellbeing' (SEWB) is preferred by many Indigenous people to 'mental health', as it implies a holistic, strengths-based perspective of mental health. ${ }^{7}$ SEWB is an important aspect of health, and the SEWB of Australia's Indigenous people is reported to be poor compared with the non-Indigenous population. ${ }^{8}$ Culturally appropriate, evidence based research strategies are needed to effectively improve the SEWB of Indigenous people.

As the health system's 'front line', primary health care services often provide SEWB-related care, including screening, early intervention and management. The stigma associated with seeking help for SEWB-related issues ${ }^{9}$ and the perception by some people that hospitals are unwelcoming ${ }^{10}$ may hinder access to mainstream mental health and state-run services. Primary health care services offer a discreet and independent alternative, and these services are often where Indigenous-focused SEWB research is conducted. This research is commonly conducted by teams that include primary health care staff, community members and researchers external to the community. Primary health care research is a challenging and resource-intensive process ${ }^{11}$, and Indigenous-focused primary health care research must also comply with Values and ethics ${ }^{3}$ and be acceptable to the community.

In this review, we aim to identify the study designs, processes, outcomes and quality indicators of Indigenous-focused SEWB primary health care research conducted by teams that include researchers who are located outside the community. A subsequent review will describe actions relating to Values and ethics ${ }^{3}$ and local protocols.

\section{Methods}

A protocol for this review has been published previously ${ }^{12}$, and is in accordance with PRISMA and MOOSE guidelines. This study is registered with PROSPERO (CRD42015024994). Database searches were conducted in Medline, Embase, CINAHL, Informit databases and HealthInfoNet, an Indigenous-specific research and project website, using the following terms: 'Indigenous', 'social and emotional wellbeing', 'mental health' and 'primary health care'. To capture studies conducted since the development of Values and ethics ${ }^{3}$, a date limit was applied from January 2003 to February 2015.

Published studies were included if they used qualitative, quantitative or mixed methods, focused on Indigenous SEWB, and were conducted in one or more primary health care services. Journal articles, reports and evaluations were included. We included studies involving research teams, including primary health care staff, community members and researchers located outside the community. SEWB describes a strengths-based holistic perspective of mental health that acknowledges the sociohistorical and personal influences on mental health. ${ }^{13}$ We included SEWB/mental health, depression disorders, anxiety disorders, dual diagnosis (SEWB and drug or alcohol use), and smoking and alcohol use.

We excluded studies that did not generate original data or where at least half the research occurred outside a primary health care service. Primary health care services included Aboriginal Medical Services $(A M S s)^{13}$, 
Aboriginal community controlled health services, and health services that provide primary health care or had general practitioners as staff members. In this review, the term AMS includes Aboriginal community controlled health services and Indigenous health services. 'Community' refers to primary health care or AMS staff, patients, families or community members. For the purpose of this review, Indigenous refers to Australian Aboriginal and/or Torres Strait Islander people.

One reviewer screened titles and abstracts, and excluded obviously irrelevant studies and duplicates. Two reviewers examined full-text versions of the articles remaining after screening. Data were extracted using data extraction forms developed for this review. In accordance with our protocol ${ }^{12}$, we assessed risk of bias using adapted versions of existing risk-of-bias assessment tools. Studies using mixed methods were assessed according to the dominant method used To assess community acceptance, we considered common aspects described in key Indigenous research documents ${ }^{3,14}$, and identified if the following criteria were reported: 1) community governance; 2) community representation in study development; 3) community representation in study conduct (data collection, data analysis); and 4) community representation in reporting. Refer to the supplementary tables (available from: www.researchgate.net/publication/317099307 FINAL_2017_05_25_Farnbach_Systematic_Review_ Supp_Tables) for details of the community acceptance assessment. We considered studies meeting three or four of the criteria as acceptable.

\section{Results}

A total of 2288 articles and program reports were identified (Figure 1). We removed 402 duplicates and excluded 1491 studies based on their title or abstract. There were 395 studies that required full-text assessment. To ensure all relevant articles and program reports were identified, we attempted to contact 50 authors to request additional data. Of these, 36 replied and 24 provided new data. A total of 37 articles relating to 25 studies were included in the review.

Multiple articles that reported findings relating to the same study (such as one evaluation ${ }^{15,16}$, one project ${ }^{17-20}$, one survey ${ }^{21,22}$, one interview/focus group session ${ }^{23-26}$, or one questionnaire ${ }^{27,28}$ ) were considered as a single study, and all references were included. The included studies focused on:

- SEWB (nine studies) ${ }^{19,20,25-34}$

- Alcohol misuse (five studies) 21,22,35-38

- Smoking cessation (four studies)23,24,39-41

- Dual diagnosis - SEWB and drug or alcohol misuse (three studies) $^{17,18,42,43}$

- Depression ${ }^{44,45}$, depression or anxiety ${ }^{46}$, or a mental health worker program (four studies). ${ }^{15,16}$

Nineteen studies were conducted in AMSs 17,18,21,22,2529,32-45, four in services aimed at providing primary health care ${ }^{19,20,23,24,30,31}$ and one in a service where general practitioners were staff members. ${ }^{15}$ One study involved community organisations, but most of the research appeared to take place in primary health care services or AMSs. ${ }^{46}$ See Supplementary Table 1 (available from: www.researchgate.net/publication/317099307_ FINAL_2017_05_25_Farnbach_Systematic_Review_ Supp_Tables) for a reference list and descriptions.

\section{Study design}

Qualitative methods were used in 18 studies $^{15-20,23-26,29-32,34,35,37,39,41-44,46}$, six of which used mixed methods $17-20,30,31,37$ and one of which was a quasiexperimental design. ${ }^{34}$ Quantitative methods on their own were used in five studies. ${ }^{21,22,27,28,33,36,45}$ One case study was included. ${ }^{40}$ Participatory action research principles were used in combination with yarning techniques ${ }^{41}$, a social-ecological perspective $e^{23,24}$ or as part of a mixed-

Figure 1. PRISMA diagram of the systematic review process

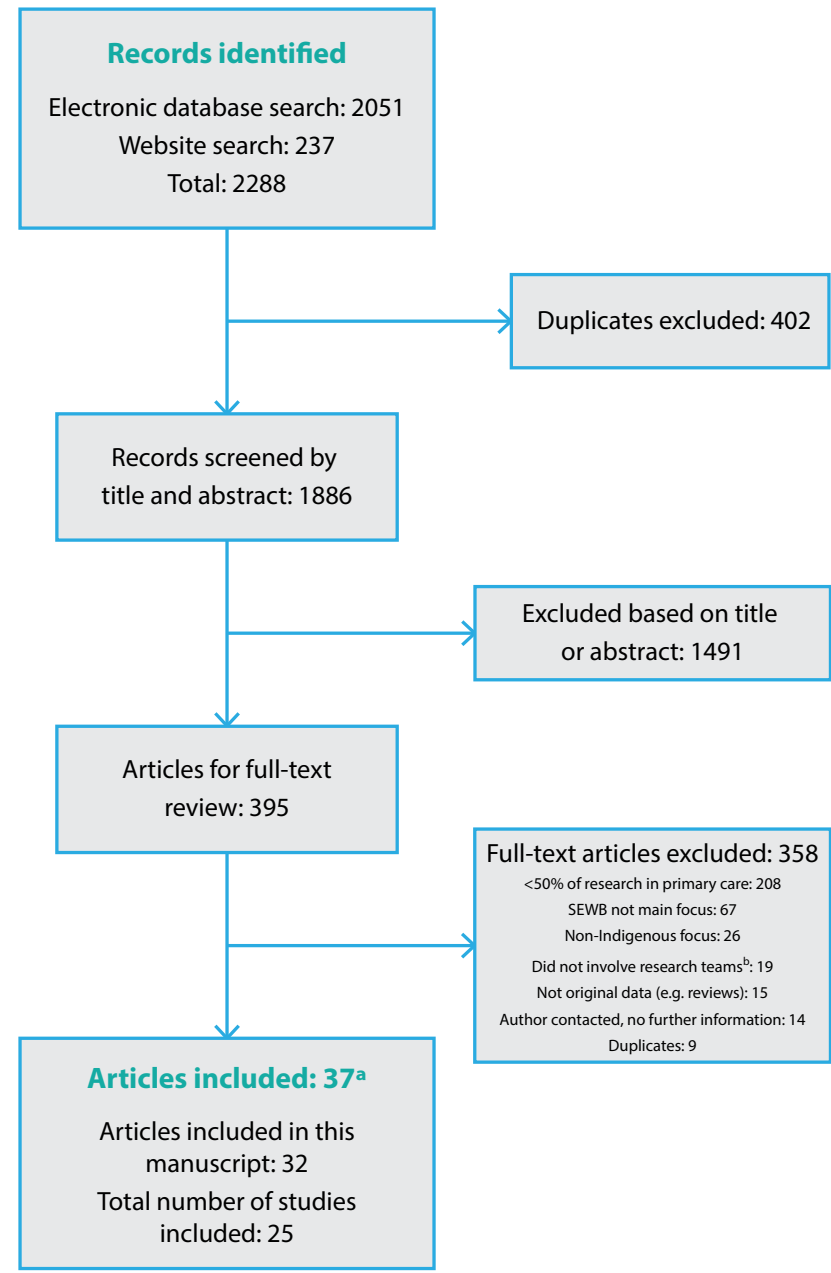

SEWB = social and emotional wellbeing

a Five articles related to studies included in the review are not referenced in this article. Details of these articles are provided in the full reference list in the supplementary tables.

b Research teams: including primary health care staff, community members and researchers located outside the community 
methods study. ${ }^{19,20}$ Two-way learning approaches were described by three studies. ${ }^{15,16,19,20,23,24}$ Sociological action research principles were used once. ${ }^{42}$ One study involved a review of existing case management models, followed by a staff survey and training. ${ }^{38}$

Three studies were part of the Australian Integrated Mental Health Initiative (AIMhi). ${ }^{19,20,3032}$ AIMhi aimed to improve outcomes for Indigenous clients of remote mental health services. AIMhi 1 developed a mental health 'brief intervention' and conducted a randomised controlled trial to evaluate the intervention. ${ }^{19,20}$ AIMhi 2 used mixed methods to examine service-level challenges ${ }^{30}$, and AIMhi 3 developed and assessed an electronic mental health resource. ${ }^{31}$ AlMhi was followed by the AlMhi Priority Driven Research Partnership, which involved the community, AMS and external researchers. ${ }^{32}$

Voices United for Harmony constituted three substudies $27,28,33,34$ to develop and assess the effectiveness of a participatory singing program to improve SEWB and physical health. AMS staff coordinated the studies' activities and participants were AMS patients. Three related studies used qualitative, mixed-methods and quantitative designs to examine staff practices ${ }^{35}$ and experiences ${ }^{37}$, and quantify the effect of staff training on alcohol screening and brief interventions in AMSs. ${ }^{36}$

Primary health care staff and patients were the most common participants. In four studies, families ${ }^{29,43}$ and carers ${ }^{19,20}$ of primary health care patients were participants. Community elders, families and residents were involved in the establishment of the AIMhi Priority Driven Research Partnership. ${ }^{32}$ Voices United for Harmony involved community leaders during study design and implementation. ${ }^{27,28,33,34}$

\section{Study initiation process}

Seven studies appeared to be initiated by researchers external to the primary health care service ${ }^{23,24,27,28,33-37}$, seven arose from research partnerships ${ }^{15,16,19,20,30-32,38,46}$ and three appeared to be jointly initiated. ${ }^{25,26,40,42} \mathrm{~A}$ community also invited a researcher from outside the community to evaluate a SEWB service. ${ }^{29}$ It was unclear how the remaining seven studies were initiated. ${ }^{17,18,21,22,39,41,43-45}$

\section{Study outcomes}

Primary outcomes were identified and met in 16 studies. ${ }^{19-29,31,33-37,39,42,43,45}$ Outcomes related to identifying participant perceptions and experiences $23,24,26,34,35,39,42,43$; evaluating an intervention ${ }^{19,20,27,28,33,34,36}$, a service ${ }^{29}$ or training ${ }^{37}$; and developing and assessing the acceptability of a resource. ${ }^{25,31}$

Two primary outcomes were identified and met in three studies. ${ }^{21-26}$ For example, one study assessed the acceptability of an alcohol-related intervention ${ }^{21}$ and identified cut-off scores of an alcohol dependence screening tool for Indigenous clients. ${ }^{22}$ Two of these articles reported on data that appear to have been collected at one time point. ${ }^{25,26}$

We were unable to identify primary outcomes in eight studies. These included a case study ${ }^{40}$; AIMhi 230; the AIMhi Priority Driven Research Partnership ${ }^{32}$; a study to develop and assess a psychological assessment tool ${ }^{44}$; and projects focused on depression ${ }^{46}$, a case management model ${ }^{38}$ and capacity development relating to dual diagnosis. ${ }^{17,18}$ The primary outcome was somewhat met in one study, where a workplace policy was developed as planned, but acceptability testing was pending. ${ }^{41}$ The AIMhi 1 and Voices United for Harmony evaluations demonstrated improved outcomes for participants who received the intervention. ${ }^{19,20,27,28,33,34}$

\section{Risk of bias and community acceptance}

We included peer-reviewed journal articles and articles from other publications describing processes, including an evaluation, description of a partnership and development of a model. Consultation, training and project reports were also included. Four studies did not align with the standard risk-of-bias assessment tools and therefore could not be assessed. ${ }^{15-18,32,38}$ In 12 studies, the risk of bias was judged to be high $^{19,20,27,28,30,33-35,37,46}$ or unclear21,22,36,40,45 , four were at moderate risk of bias ${ }^{19,20,25,26,41,42,44}$ and five were at a low risk of bias. ${ }^{23,24,29,31,39,43}$ AIMhi 1 was assessed using the qualitative ${ }^{19}$ and randomised controlled trial ${ }^{20}$ risk-of-bias tools, because these methods were reported separately.

Using the qualitative risk-of-bias tool, most studies were deemed to have a moderate $25,26,41,42,44$ or high $^{19,20,30,35,37,46}$ risk of bias. These ratings were because of missing or unclear reporting of many of the criteria used to assess bias. For example, actions related to ethical issues were not reported in more than half the studies. Processes related to informed consent, confidentiality or consideration of the impact of the authors' relationship with the participants were described by authors of six studies. ${ }^{23,24,29,31,39,42,43}$

Assessment of quantitative studies presented specific challenges. Quantitative studies included a survey ${ }^{21,22}$, quality improvement cohort study ${ }^{36}$, case study ${ }^{40}$, validation study ${ }^{45}$ and cohort analytic quasi-experimental design. ${ }^{27,28,33,34}$ Many assessment criteria were not applicable for these designs. For example, assessing intervention integrity was not applicable when assessing the validation study ${ }^{45}$ or the quality improvement cohort study. ${ }^{36}$

The Voices United for Harmony quasi-experimental designs ${ }^{27,28,33,34}$ were found to have high risk of bias because of participant self-selection (selection bias), confounding, lack of blinding and the high number of dropouts in two of the studies. ${ }^{33,34}$ In these studies and the case study, participant selection methods were developed in response to community feedback and were based on participants' ability or willingness to take part in the program or intervention, rather than using sampling, 
randomised or consecutive methods. 27,28,33,34,40 Two of the Voices United for Harmony initiatives used valid and reliable data collection tools ${ }^{27,28,33}$, leading to a low risk of bias for this criteria.

The AIMhi 1 randomised controlled trial was judged to have a moderate risk of bias. ${ }^{20}$ Documentation of allocation concealment and blinding of participants and personnel during outcome assessment were unclear.

Communities' perspectives were rarely reported. However, nine studies were assessed as having high community acceptance (three to four criteria met) $)^{21-24,27,28,32,39,41,44-46}$, and 12 as low acceptance (two

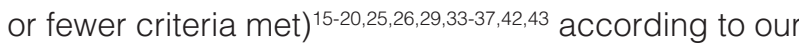
four criteria. Four studies were part of large, ongoing research partnerships, which may have involved extensive community engagement, but this was not described. 19,20,25,26,30,31 We were unable to determine community acceptance in four studies. ${ }^{30,31,38,40}$ This was because of the lack of reporting between linked articles ${ }^{30,31}$ or because primary health care service staff were co-authors and the extent of their involvement during research development, conduct and reporting was not described. ${ }^{38,40}$ Two studies were judged as having high community acceptance and low risk of bias. ${ }^{23,24,39}$

\section{Discussion}

We identified only two studies that were judged to be scientifically robust and acceptable to the community. ${ }^{23,24,39}$ Other studies with high community acceptance were deemed to have a moderate ${ }^{41,44}$, unclear or high risk of bias $21,22,27,28,45,46$, or were unable to be assessed ${ }^{32}$ using standard assessment tools. This results in uncertainty about the strength and generalisability of their findings. Where community perspectives were unclear, it was difficult to determine if this was because of underreporting (possibly related to publication word limits) or if it reflected community dissatisfaction. Although not explicitly reported, involving community members in key positions or extensive community consultation may suggest acceptance and have led to culturally appropriate designs.

A variety of designs and processes were used in the included studies. These depended on the study aim, the collaboration and the community involved. This variation, together with the diversity among Indigenous communities, makes drawing general conclusions about designs challenging.

Qualitative studies appeared to have greater community acceptance and lower risk of bias than quantitative and mixed-methods studies. However, qualitative research is considered Level IV evidence in the scientific community, meaning there is a lack of certainty when drawing conclusions from its findings. In addition, the primary outcomes identified in most of the qualitative studies involved identifying perceptions or experiences, suggesting limited impact on primary health care delivery.

Concerns about randomised controlled trials that involve Indigenous communities include the perception that randomisation is unethical. ${ }^{47}$ However, randomised controlled trials are considered Level I evidence in the scientific community. Two studies in this review involved randomised controlled trials, and both used flexible randomisation processes. In one ${ }^{19,20}$, participants were randomised into 'early' and 'late' intervention groups, meaning that all participants received the intervention at different time points. Although the authors did not provide justification for this approach, the study was part of a large, ongoing initiative, suggesting collaboration with the community. In the other study, the design was modified to a nonrandomised, quasi-experimental design in response to community feedback. ${ }^{27,28}$ Both studies were assessed as having a high risk of bias, demonstrating the challenges of implementing study designs in Indigenous communities that are considered high quality in the academic community.

These challenges surrounding randomised controlled trials have been reported previously, including by the authors of one study ${ }^{48}$, who described modifying the design to address challenges and encourage recruitment. The researchers ceased this study, citing clinic, patient, staff and study design-related factors that made the project untenable. Evidence based research methods have developed within a Western cultural perspective, which does not incorporate Indigenous social, cultural or historical perspectives. These examples demonstrate how evidence based research methods may not be appropriate for Indigenous communities, because of these differing perspectives.

Culturally sensitive approaches, including two-way learning 15,16,19,20,23,24, participatory ${ }^{19,20,23,24,41}$, social-ecological ${ }^{23,24}$ and phenomenological approaches ${ }^{39}$ were used in five studies. In one, participatory action research was used to localise an intervention and study design. ${ }^{19,20}$ These approaches appeared to improve community acceptance by incorporating local perspectives. We propose that research projects incorporate these culturally sensitive approaches, as identified in this review.

There is increasing focus on methods that incorporate Indigenous perspectives and Indigenous ways of knowing, being and doing. ${ }^{49}$ Regardless of the topic under investigation, research incorporating Indigenous perspectives will lead to primary health care that is better aligned with the needs of Indigenous people. However, there appear to be few examples of their implementation in practice. ${ }^{50}$

In this review, we identified outcomes related to evaluating interventions, services or training in seven studies $^{19,20,2729,33,34,36,37}$ or assessing resources in two studies. ${ }^{25,31}$ These outcomes indicate potential impact at the community level. Research should improve health 
and result in community-level benefit. ${ }^{2}$ We propose that research is reported according to the following outcomes:

1. Process outcomes that describe steps taken during planning and implementation

2. Academic outcomes that describe dissemination and academic achievements

3. Clinical outcomes that describe efficacy, impact, costeffectiveness and research translation

4. Community outcomes that describe ongoing implementation and efficacy, cost savings, access changes, community engagement and other outcomes that the community determines to be relevant.

Reporting these outcomes will provide a balanced description of how to achieve high-quality, communityendorsed research that is likely to affect clinical practice and health outcomes. We suggest considering these outcomes, together with the community-acceptance principles highlighted in this review, when assessing the quality of Indigenous-focused research.

There are several limitations to this review. The breadth of formats included (evaluations, reports and journal articles) did not fit easily with standard risk-ofbias assessment tools, and we modified these tools to make assessment feasible. We were restricted to the information reported in articles, which may exclude some information. Although we have identified criteria to indicate community involvement and acceptance, we recognise this may not comprehensively capture all aspects of culturally appropriate research. In addition, we recognise that the diversity of Indigenous communities throughout Australia means that a process that is suitable in one community may not be suitable in another.

\section{Conclusion}

There are few examples of Indigenous-focused SEWB primary health care research that are of high scientific quality and acceptable to the community. This provides many opportunities for improvements for research in all domains. Use of participatory action research, social-ecological approaches and incorporation of two-way learning principles appears to facilitate research that incorporates Indigenous perspectives. We recommend that consideration of community-level outcomes and the community-acceptance principles highlighted in this review are kept at the forefront throughout research. This will improve culturally appropriate research that positively impacts the SEWB of Indigenous people.

\section{Acknowledgements}

We acknowledge staff at the University of Sydney Medical Library for their help developing the search strategy. AE was in receipt of a National Health and Medical Research Scholarship (APP1056434). JF was in receipt of the Rowan Nicks Russell Drysdale Fellowship and $\mathrm{MH}$ was in receipt of a National Heart Foundation Future Leader Fellowship (100034). These funding bodies had no role in the conduct or reporting of this review.

\section{Competing interests}

None declared

\section{Author contributions}

SF led this review. AE was the second reviewer for the data extraction and analysis. MH supervises SF and $A E$ and provided oversight to this review. NG helped to develop the study protocol and reporting. JF and $J G$ provided input on the discussion and conclusions. All authors reviewed and contributed to writing the manuscript.

\section{References}

1. Kendall E, Sunderland N, Barnett L, Nalder G, Matthews $\mathrm{C}$. Beyond the rhetoric of participatory research in Indigenous communities: advances in Australia over the last decade. Qual Health Res. 2011;21(12):1719-28.

2. Bainbridge R, Tsey K, McCalman J, Kinchin I, Saunders V, Lui F, et al. No one's discussing the elephant in the room: contemplating questions of research impact and benefit in Aboriginal and Torres Strait Islander Australian health research. BMC Public Health. 2015;15(1):696.

3. National Health and Medical Research Council. Values and ethics: guidelines for ethical conduct in Aboriginal and Torres Strait Islander health research. Canberra: Commonwealth of Australia; 2003 [cited 2014 Sep 1]. Available from: www.nhmrc.gov.au/_files_nhmrc/ publications/attachments/e52.pdf

4. Dunbar T, Arnott A, Scrimgeour M, Henry J, MurakamiGold L. Working towards change in Indigenous health research. Darwin: Cooperative Research Centre for Aboriginal Health; 2004 [cited 2017 May 15]. Available from: www.lowitja.org.au/sites/default/files/docs/ LinksReport.pdf

5. Elliott E, Latimer J, Fitzpatrick J, Oscar J, Carter M. There's hope in the valley. J Paediatr Child Health. 2012;48(3):190-2.

6. Wilson S. Research is ceremony: Indigenous research methods. Black Point, NS: Fernwood Publishing; 2008.

7. Garvey D. A review of the social and emotional wellbeing of Indigenous Australian peoples. Perth: Australian Indigenous HealthInfoNet; 2008 [cited 2015 Jul 3]. Available from: www.healthinfonet.ecu.edu.au/uploads/ docs/sewb-review.pdf 
8. Australian Bureau of Statistics. Australian Aboriginal and Torres Strait Islander health survey: first results, Australia, 2012-13. Canberra: Australian Bureau of Statistics; 2013 [cited 2014 Sep 23]. Available from: www.abs.gov.au/ ausstats/abs@.nsf/Latestproducts/9F3C9BDE98B3C5F1C A257C2F00145721?opendocument

9. Isaacs AN, Pyett P, Oakley-Browne MA, Gruis H, WaplesCrowe $P$. Barriers and facilitators to the utilization of adult mental health services by Australia's Indigenous people: seeking a way forward. Int J Ment Health Nurs. 2010;19(2):75-82.

10. Durey A, Wynaden D, Thompson SC, Davidson PM, Bessarab D, Katzenellenbogen JM. Owning solutions: a collaborative model to improve quality in hospital care for Aboriginal Australians. Nurs Inq. 2012;19(2):144-52.

11. Yallop J, McAvoy B, Croucher J, Tonkin A, Piterman L. Primary health care research - essential but disadvantaged. Med J Aust. 2006;185(2):118-20.

12. Farnbach S, Eades AM, Hackett M. Australian Aboriginal and Torres Strait Islander-focused primary healthcare social and emotional wellbeing research: a systematic review protocol. Syst Rev. 2015;4(189).

13. National Australian Community Controlled Health Organisation. Canberra: NACCHO; 2016. Definitions; 2014 [cited 2015 Oct 19]; [about 4 screens]. Available from: www.naccho.org.au/aboriginal-health/definitions

14. Arabena K, Moodie D. The Lowitja Institute: building a national strategic research agenda to improve the health of Aboriginal and Torres Strait Islander peoplesresearch enterprise. Med J Aust. 2011;194(10):532-4.

15. Harris A, Robinson G. The Aboriginal Mental Health Worker Program: the challenge of supporting Aboriginal involvement in mental health care in the remote community context. Australian e-Journal for the Advancement of Mental Health. 2007;6(1):11

16. Robinson G, Harris A. Aboriginal Mental Health Worker Program: final evaluation report. Darwin: School for Social and Policy Research, Institute of Advanced Studies, Charles Darwin University; 2004 [cited 2017 Jun 2]. Available from: nceta.flinders.edu.au/ files/6813/5787/2431/Abor_mh_worker_program_final.pdf

17. Bakos K, Victorian Dual Diagnosis Education and Training Unit. Indigenous dual diagnosis training project stage 1: the consultation report. Melbourne: Victorian Dual Diagnosis Education and Training Unit; 2008. Copy available from author.

18. Bakos K, Logan G. Indigenous dual diagnosis training project stage 3: training evaluation report. Melbourne: Victorian Dual Diagnosis Initiative, St Vincent's Health Australia. Copy available from author.

19. Nagel T, Thompson C, Robinson G, Trauer T, Condon J. Two-way approaches to Indigenous mental health literacy. Aust J Prim Health. 2009;15(1):50-5.
20. Nagel T, Robinson G, Condon J, Trauer T. Approach to treatment of mental illness and substance dependence in remote Indigenous communities: results of a mixed methods study. Aust J Rural Health. 2009;17(4):174-82.

21. Calabria B, Clifford A, Shakeshaft A, Allan J, Bliss D, Doran C. The acceptability to Aboriginal Australians of a family-based intervention to reduce alcohol-related harms. Drug Alcohol Rev. 2013;32(3):328-32.

22. Calabria B, Clifford A, Shakeshaft A, Conigrave K, Simpson L, Bliss D, et al. Identifying Aboriginal-specific AUDIT-C and AUDIT-3 cutoff scores for at-risk, highrisk, and likely dependent drinkers using measures of agreement with the 10-item Alcohol Use Disorders Identification Test. Addict Sci Clin Pract. 2014;9:17.

23. Dawson AP, Cargo M, Stewart H, Chong A, Daniel M. Aboriginal health workers experience multilevel barriers to quitting smoking: a qualitative study. Int J Equity Health. 2012;11:27.

24. Dawson AP, Cargo M, Stewart H, Chong A, Daniel M. "I know it's bad for me and yet I do it": exploring the factors that perpetuate smoking in Aboriginal health workers - a qualitative study. BMC Health Serv Res. 2012;12:102.

25. Williamson A, Redman S, Dadds M, Daniels J, D'Este C, Raphael B, et al. Acceptability of an emotional and behavioural screening tool for children in Aboriginal Community Controlled Health Services in urban NSW. Aust N Z J Psychiatry. 2010;44(10):894-900.

26. Williamson AB, Raphael B, Redman S, Daniels J, Eades SJ, Mayers N. Emerging themes in Aboriginal child and adolescent mental health: findings from a qualitative study in Sydney, New South Wales. Med J Aust. 2010;192(10):603-5.

27. Sun J, Buys N. Effectiveness of a participative community singing program to improve health behaviors and increase physical activity in Australian Aboriginal and Torres Strait Islander people. Int J Disabil Hum Dev. 2013;12(3):297-304.

28. Sun J, Buys N. Effects of community singing program on mental health outcomes of Australian Aboriginal and Torres Strait Islander people: a meditative approach. Am J Health Promot. 2016;30(4):259-63.

29. Carey TA. A qualitative study of a social and emotional well-being service for a remote Indigenous Australian community: implications for access, effectiveness, and sustainability. BMC Health Serv Res. 2013;13:80.

30. Nagel T. The need for relapse prevention strategies in Top End remote Indigenous mental health. Australian e-Journal for the Advancement of Mental Health. 2006;5(1):1-5.

31. Dingwall K, Puszka S, Sweet M, Nagel T. "Like drawing into sand": acceptability, feasibility, and appropriateness of a new e-mental health resource for service providers working with Aboriginal and Torres Strait Islander people. Aust Psychologist. 2012;50(1):60-9. 
32. Haswell-Elkins M, Reilly L, Fagan R, Ypinazar V, Hunter E, Tsey K, et al. Listening, sharing understanding and facilitating consumer, family and community empowerment through a priority driven partnership in Far North Queensland. Australas Psychiatry. 2009;17 Suppl(2009):S54-8.

33. Sun J, Buys N. Participatory community singing program to enhance quality of life and social and emotional wellbeing in Aboriginal and Torres Strait Islander Australians with chronic diseases. Int Journal Disabil Hum Dev. 2013;12(3):317-23.

34. Sun J, Buys N. Improving Aboriginal and Torres Strait Islander Australians' well-being using participatory community singing approach. Int J Disabil Hum Dev. 2013;12(3):305-16.

35. Clifford A, Shakeshaft A, Deans C. How and when healthcare practitioners in Aboriginal Community Controlled Health Services deliver alcohol screening and brief intervention, and why they don't: a qualitative study. Drug Alcohol Rev. 2012;31(1):13-9.

36. Clifford A, Shakeshaft A, Deans C. Training and tailored outreach support to improve alcohol screening and brief intervention in Aboriginal Community Controlled Health Services. Drug Alcohol Rev. 2013;32(1):72-9.

37. Clifford A, Shakeshaft A. Evidence-based alcohol screening and brief intervention in Aboriginal Community Controlled Health Services: experiences of health-care providers. Drug Alcohol Rev. 2011;30(1):55-62.

38. Lovett R, Dance P, Guthrie J, Brown R, Tongs J. Walan Girri: developing a culturally mediated case management model for problematic alcohol use among urban Indigenous people. Aust Health Rev. 2014;38(4):440-6.

39. Bond C, Brough M, Spurling G, Hayman N. 'It had to be my choice' Indigenous smoking cessation and negotiations of risk, resistance and resilience. Health Risk Soc. 2012;14(6):565-81.

40. DiGiacomo M, Davidson PM, Davison J, Moore L, Abbott P. Stressful life events, resources, and access: key considerations in quitting smoking at an Aboriginal Medical Service. Aust N Z J Public Health. 2007;31(2):174-6.

41. Fletcher G, Fredericks B, Adams K, Finlay S, Andy S, Briggs L, Hall R. Having a yarn about smoking: using action research to develop a 'no smoking' policy within an Aboriginal Health Organisation. Health Policy. 2011;103(1):92-7.
42. Allan J. Engaging primary health care workers in drug and alcohol and mental health interventions: challenges for service delivery in rural and remote Australia. Aust J Prim Health. 2010;16(4):311-8.

43. Lee KS, Harrison K, Mills K, Conigrave KM. Needs of Aboriginal Australian women with comorbid mental and alcohol and other drug use disorders. Drug Alcohol Rev. 2014;33(5):473-81.

44. Esler D, Johnston F, Thomas D. The acceptability of a depression screening tool in an urban, Aboriginal community-controlled health service. Aust N Z J Public Health. 2007;31(3):259-63.

45. Esler D, Johnston F, Thomas D, Davis B. The validity of a depression screening tool modified for use with Aboriginal and Torres Strait Islander people. Aust N Z J Public Health. 2008;32(4):317-21.

46. Higgins R, Murphy B, Jobling K. Improving the wellbeing of Melbourne-based Aboriginal people with chronic disease and experiencing depression, anxiety or a related mental health disorder: a Wurundjeri community driven initiative. Melbourne: Heart Research Institute, The University of Melbourne, Wurundjeri Tribe; 2013 [cited 2017 Jun 2]. Available from: www.beyondblue.org. au/docs/default-source/research-project-files/bw0165. pdf?sfvrsn=2

47. Carson K, Smith B. Methodological challenges and options for addressing them in Aboriginal and Torres Strait Islander health research. Australas Epidemiologist. 2014;21(2):47-50.

48. Sibthorpe BM, Bailie RS, Brady MA, Ball SA, SumnerDodd P, Hall WD. The demise of a planned randomised controlled trial in an urban Aboriginal medical service. Med J Aust. 2002;176(6):273-6.

49. Martin K. Ways of knowing, ways of being, ways of doing: Developing a theoretical framework and methods for Indigenous re-search and Indigenist research. J Aust Studies. 2001;76:203-14.

50. Mc Loughlin F, Hadgraft NT, Atkinson D, Marley JV. Aboriginal health research in the remote Kimberley: an exploration of perceptions, attitudes and concerns of stakeholders. BMC Health Serv Res. 2014;14(1):517.

\section{Copyright: (c) (i) (2)}

(c) 2017 Farnbach et al. This article is licensed under the Creative Commons Attribution-NonCommercial-ShareAlike 4.0 International Licence, which allows others to redistribute, adapt and share this work non-commercially provided they attribute the work and any adapted version of it is distributed under the same Creative Commons licence terms. See: www.creativecommons.org/licenses/by-nc-sa/4.0/ 